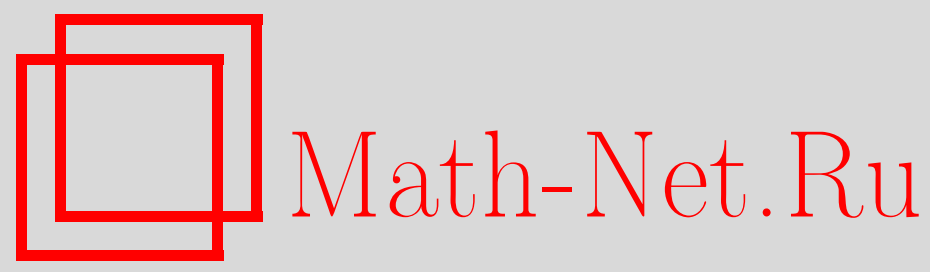

И. В. Басов, О разрешимости уравнений нелинейной идеально-вязкой сжимаемой жидкости, Матем. заметки, 2000, том 68, выпуск 1, 13-23

DOI: https://doi.org/10.4213/mzm915

Использование Общероссийского математического портала Math-Net.Ru подразумевает, что вы прочитали и согласны с пользовательским соглашением http://www.mathnet.ru/rus/agreement

Параметры загрузки:

IP : 3.91 .87 .62

26 апреля 2023 г., 18:35:42

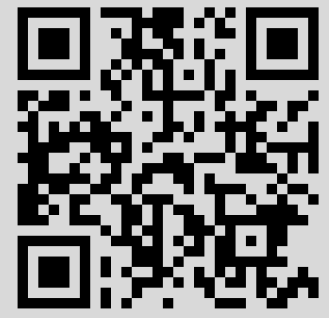




\title{
О РАЗРЕШИМОСТИ УРАВНЕНИЙ НЕЛИНЕЙНОЙ ИДЕАЛЬНО-ВЯЗКОЙ СЖКИАЕМОЙ ЖИДКОСТИ
}

\author{
И.В. Басов
}

Рассматривается модель одномерных движений вязкой сжимаемой жидкости. Описывается некоторый класс нелинейных законов напряженного состояния, для которых соответствующая начально-краевая задача разрешима в целом по времени в классе обобщенных решений, удовлетворяющих энергетическому тождеству. В частности, исследуются среды, проявляюшие вязкие свойства лишь при больших скоростях деформаций.

Библиографоия: 11 названий.

1. Введение. Рассмотрим систему уравнений, описывающую одномерные вертикальные движения горизонтальных слоев жидкости с нелинейньм законом зависимости тензора напряжений от тензора скоростей деформаций. Будем считать, что движение происходит вдоль пространственной координаты $x$, лежащей на отрезке $\Omega=[l, r]$, со скоростью $u(x, t)$. В случае баротропного движения с давлением $p$, пропорциональным плотности $\rho(x, t)$ ( $p=R \rho$, где $R>0$ константа), и отсутствия внешних сил уравнения движения имеют вид [1]

$$
\begin{gathered}
\rho_{t}+(\rho u)_{x}=0 \\
\rho\left(u_{t}+u u_{x}\right)=\sigma_{x}-R \rho_{x}
\end{gathered}
$$

где $(x, t) \in Q_{T}=\Omega \times(0, T)$. Здесь $\sigma=f\left(u_{x}\right)$ - неравновесная часть напряжений, отвечающая за вязкость (диссипацию энергии). К этим уравнениям добавляются начальные

$$
\rho=\rho_{0}, \quad u=u_{0} \text { при } t=0
$$

и краевые условия

$$
u=0 \quad \text { при } \quad x \in \Gamma=\partial \Omega=\{l, r\} .
$$

Достаточно хорошо исследован случай, когда зависимость $\sigma$ от $u_{x}$ (далее, для краткости будем писать $s$ вместо $u_{x}$ ) линейная, в том числе когда $\sigma$ имеет вид $\sigma=h(\rho) u_{x}$ (см. [2]-[4]). В частности, в [5] доказана разрешимость многомерных задач. Среди работ по системам с нелинейным законом напряженного состояния следует отметить [6], [7].

Исследования проведены при частичной поддержке Российского фонда фундаментальных исследований, грант № 96-01-01524. 
Цель данной работы - описание некоторого класса законов напряженного состояния, в том числе с участками постоянства, для которых задача (1)-(4) имеет слабые обобщенные решения в целом по времени. Допускается, например, следующая зависимость:

$$
f(s)= \begin{cases}\nu\left(s+s_{0}\right), & s \leqslant-s_{0} \\ 0, & |s| \leqslant s_{0} \\ \nu\left(s-s_{0}\right), & s_{0} \leqslant s .\end{cases}
$$

Жидкости с такими законами напряженного состояния при малых скоростях деформаций являются идеальными, а при возрастании $\left|u_{x}\right|$ в них проявляются вязкие свойства. Иначе говоря, данная модель соответствует сплошньм средам со смешанными газо-жидкостными свойствами [8].

Кроме того, в работе строятся решения для задач с разрывными законами напряженного состояния.

2. Постановка задачи и основной результат. Для точной формулировки условий на функцию $f(s)$ удобно ввести ее первообразную

$$
F(s)=\int_{0}^{s} f(s) d s .
$$

Предполагая $f(s)$ неубывающей функцией от $s$, заметим, что $F(s)$ является (нестрого) выпуклой и непрерьвной. Обозначим через $\partial F(s)$ субдифференциал $F(s)$, определяемый равенством

$$
\partial F(s)=\{a \in \mathbb{R} \mid a(t-s) \leqslant F(t)-F(s) \forall t \in \mathbb{R}\} ;
$$

$\partial F$ можно назвать многозначным отображением, так как для любого $s \in \mathbb{R}$ значение $\partial F(s)$ является, вообше говоря, подмножеством $\mathbb{R}$, т. е. $\partial F(s): \mathbb{R} \rightarrow 2^{\mathbb{R}}$. При этом для любого $s \in \mathbb{R}$ значение функции $f(s)$ является элементом множества $\partial F(s)$, и закон напряженного состояния записывается в виде

$$
\sigma \in \partial F(s)
$$

В настоящей работе исследуется случай, когда функция $F(s)$ удовлетворяет следующим условиям.

1. $F(s)$ вьпуклая функция от $s$. В частности, $\partial F(s)$ является неубывающим по $s$, т. е. $\forall s_{1}, s_{2} \in \mathbb{R}, \forall \sigma_{i} \in \partial F\left(s_{i}\right)$ вьполнено неравенство

$$
\left(\sigma_{1}-\sigma_{2}\right)\left(s_{1}-s_{2}\right) \geqslant 0 .
$$

2. Для всех $s \in \mathbb{R}$ и всех $\sigma \in \partial F(s)$ справедливо неравенство $\sigma s \geqslant 0$. (Оно следует из условия неубывания энтропии.)

3. Существуют такие константы $K>0, L>0$, что $\forall s \in \mathbb{R}, \forall \sigma \in \partial F(s)$ имеют место неравенства $K s-L \leqslant \sigma \leqslant K s+L$.

4. Для произвольного малого $\delta>0$ существует дважды дифференцируемая монотонная функция $F^{\delta}(s)$, удовлетворяющая свойствам 1-3 с теми же константами $L$ и $K$, такая, что $\left|F(s)-F^{\delta}(s)\right| \leqslant M \delta$, где $M$ не зависит от $\delta$, и

$$
\left(F^{\delta}(s)\right)^{\prime \prime} \geqslant \delta \text {. }
$$

Из свойств функции $F^{\delta}(s)$ следует, что $\partial F^{\delta}(s)$ является однозначным отображением, и, обозначая $f^{\delta}(s)=\partial F^{\delta}(s)$, можно записать условия 1-3 в терминах функции $f^{\delta}(s)$. 
Для функций $f(s)$, графики которых имеют конечное число изломов, разрывов и участков, где $f^{\prime}(s) \leqslant \delta$ для всех малых $\delta$, условия $1-4$ выполняются.

ОПРЕДЕЛЕниЕ. Пара функций $(u, \rho)$ называется обобшенным решением начально-краевой задачи (1)-(5), если $\rho>0$, справедливы включения

$$
u \in L_{\infty}\left(0, T ; L_{2}(\Omega)\right) \cap L_{2}\left(0, T ; W_{2}^{1}(\Omega)\right), \quad \rho, \frac{1}{\rho} \in L_{\infty}\left(Q_{T}\right),
$$

включение (5) вьполнено почти всюду в $Q_{T}$ и для почти всех $t \in(0, T)$ и для всех функций $\varphi, \psi \in C^{\infty}\left(Q_{T}\right)$, равных нулю на $\Gamma$, справедливы интегральные тождества

$$
\begin{aligned}
\int_{0}^{t} \int_{\Omega} \rho\left(\varphi_{t}+u \varphi_{x}\right) d x d s & =\int_{\Omega} \rho \varphi d x-\int_{\Omega} \rho_{0} \varphi(x, 0) d x \\
\int_{0}^{t} \int_{\Omega}\left(\rho u \psi_{t}+\rho u u \psi_{x}+f\left(u_{x}\right) \psi_{x}-R \rho \psi_{x}\right) d x d s & =\int_{\Omega} \rho u \psi d x-\int_{\Omega} \rho_{0} u_{0} \psi(x, 0) d x .
\end{aligned}
$$

При этом $u=0$ на $\Gamma$ (как непрерьвная функция по $x$ ) для почти всех $t \in(0, T)$.

Основным результатом работы является следующая теорема.

ТЕОремА. Если функиия $f(s)$ удовлетворяет условиям $1-4, u_{0} \in L_{2}(\Omega), \rho_{0}, 1 / \rho_{0}$ $\in L_{\infty}(\Omega)$ и $\rho_{0}>0$, то существует обобщенное решение задачи (1)-(5) в смысле указанного выше определения.

Если $u_{0} \in L_{q}(\Omega)$ c $2<q<\infty$, mo $u \in L_{\infty}\left(0, T ; L_{q}(\Omega)\right)$.

3. Построение приближенных решений. В этом пункте мы докажем локальное по времени существование сильных обобщенных решений задачи, состоящей из системы уравнений

$$
\begin{gathered}
\rho_{t}+(\rho u)_{x}=0 \\
\rho\left(u_{t}+u u_{x}\right)=f^{\delta}\left(u_{x}\right)_{x}-R \rho_{x}
\end{gathered}
$$

и начально-краевых условий

$$
\begin{gathered}
\rho=\rho_{0}^{\delta}, \quad u=u_{0}^{\delta} \quad \text { при } t=0, \\
u=0 \quad \text { при } \quad x \in \Gamma,
\end{gathered}
$$

где $\rho_{0}^{\delta} \in W_{2}^{1}(\Omega), \rho>0$ и $u_{0}^{\delta} \in W_{2}^{1}(\Omega)$ таковы, что $\left\|\rho_{0}-\rho_{0}^{\delta}\right\|_{L_{2}(\Omega)}+\left\|u_{0}-u_{0}^{\delta}\right\|_{L_{2}(\Omega)} \leqslant \delta$, a $f^{\delta}(s)$ функция из условия 4 п. 2 .

Прежде всего заметим, что для любого целого $N>0$ найдется интервал времени $\left(0, t_{N}\right)$, на котором сушествует решение задачи

$$
\begin{gathered}
\rho_{t}+(\rho u)_{x}=0, \\
\int_{\Omega}\left(\rho\left(u_{t}+u u_{x}\right)-f^{\delta}\left(u_{x}\right)_{x}-R \rho_{x}\right) \omega_{j}(x) d x=0, \quad j=1, \ldots, N, \\
\rho=\rho_{0}^{\delta}(x) \quad \text { при } t=0, \\
u=\sum_{j=1}^{N} u_{j}(t) \omega_{j}(x), \quad u(x, 0)=u_{0}^{\delta N}(x),
\end{gathered}
$$


где

$$
\omega_{j}(x)=\sin \left(\frac{x-l}{r-l} \pi j\right), \quad u_{0}^{\delta N}(x)=\sum_{j=1}^{N} \frac{2 \omega_{j}(x)}{r-l} \int_{\Omega} u_{0}^{\delta}(\xi) \omega_{j}(\xi) d \xi .
$$

В изоморфном $C\left(0, t_{N}\right)^{N}$ пространстве

$$
X=\left\{u=\sum_{j=1}^{N} u_{j}(t) \omega_{j}(x) \mid u_{j}(t) \in C\left(0, t_{N}\right)\right\}
$$

с нормой, определенной равенством $\|u\|_{X}^{2}=\sup _{\left(0, t_{N}\right)} \sum_{j=1}^{N}\left|u_{j}(t)\right|^{2}$, рассмотрим множество

$$
B=\left\{u \in X \mid\|u\|_{X} \leqslant b, u(x, 0)=u_{0}^{\delta N}(x)\right\}, \quad \text { где } b^{2}=1+\frac{\sup _{\Omega} \rho_{0}^{\delta}(x)}{\inf _{\Omega} \rho_{0}^{\delta}(x)}\left\|u_{0}^{\delta N}\right\|_{L_{2}(\Omega)}^{2} .
$$

Для функции $u(x, t) \in B$ и $\rho_{0}^{\delta}(x) \in W_{2}^{1}(\Omega)$ можно построить, например, методом характеристик или методом Галёркина, функцию $\rho(x, t)$, удовлетворяющую уравнениям $(11),(13)$. Для $\rho$ справедливы включения $\rho, \rho_{x}, \rho_{t} \in L_{\infty}\left(0, t_{N} ; L_{2}(\Omega)\right)$. Кроме того, поскольку для $u \in X$ вьполняется неравенство $\left\|u_{x x}\right\|_{X} \leqslant N^{2}\|u\|_{X}$, то для $\rho$ имеется оценка

$$
e^{-N^{2} b t} \inf _{\Omega} \rho_{0}^{\delta}(x) \leqslant \rho(x, t) \leqslant e^{N^{2} b t} \sup _{\Omega} \rho_{0}^{\delta}(x) .
$$

Затем для функций $u(x, t)$ и $\rho(x, t)$ существует функция $U \in X$ вида

$$
U=\sum_{j=1}^{N} U_{j}(t) \omega_{j}(x)
$$

удовлетворяющая равенствам

$$
U(x, 0)=u(x, 0), \quad \int_{\Omega}\left(\rho\left(U_{t}+u U_{x}\right)-f^{\delta}\left(U_{x}\right)_{x}-R \rho_{x}\right) \omega_{j} d x=0, \quad j=1, \ldots, N .
$$

Действительно, $U_{j}(t)$ определяются из решения задачи Коши для системы обыкновенных дифференциальных уравнений первого порядка. Неравенство

$$
\operatorname{det} \int_{\Omega} \rho \omega_{i} \omega_{j} d x \neq 0
$$

выполненное в силу положительности $\rho$, позволяет разрешить эту систему относительно производных, что вместе с непрерывностью $f^{\delta}(s)$ обосновьвает существование функции $U$. Таким образом, определен оператор $A: B \rightarrow X$ такой, что $A(u)=U$.

Оценивая с помощью неравенства Юнга правую часть равенства

$$
\frac{d}{d t} \int_{\Omega} F^{\delta}\left(u_{x}\right) d x+\int_{\Omega} \rho U_{t}^{2} d x=\int_{\Omega}\left(-R \rho_{x} U_{t}+\rho u U_{x} U_{t}\right) d x,
$$

заключаем, что $U_{j} \in W_{2}^{1}\left(0, t_{N}\right)$ и, следовательно, $A$ компактен в $X$. Прямой проверкой доказьвается непрерьвность $A: B \rightarrow X$ (см. [2], [7], [9]).

Равенство

$$
\frac{1}{2} \frac{d}{d t} \int_{\Omega} \rho U^{2} d x+\int_{\Omega} f^{\delta}\left(U_{x}\right) U_{x} d x=-\int_{\Omega} R \rho_{x} U d x
$$

и неравенства для $\rho$ и при достаточно малых $t_{N}$, дают оценку $\|U\|_{X} \leqslant b$, равносильную условию $A(B) \subset B$. Таким образом, оператор $A$ и множество $B \subset X$ удовлетворяют условиям теоремы Шаудера. Несложно заметить, что $u$-неподвижная точка $A$-вместе с соответствуюшей функцией $\rho$ являются решением задачи (11)-(14). 
ЛЕмма 1. Для решений $(u, \rho)$ задач (11)-(14) при фиксированном $\delta>0$ существуют не зависящие от $N$ положительные константы $\tau$ и с такие, что

$$
\begin{aligned}
& \|u\|_{L_{\infty}\left(0, \tau ; W_{2}^{1}(\Omega)\right)}+\|u\|_{L_{2}\left(0, \tau ; W_{2}^{2}(\Omega)\right)}+\|\rho\|_{L_{\infty}\left(0, \tau ; W_{2}^{1}(\Omega)\right)} \\
& \quad+\left\|\rho_{t}\right\|_{L_{\infty}\left(0, \tau ; L_{2}(\Omega)\right)}+\left\|u_{t}\right\|_{L_{2}\left(Q_{\tau}\right)}+\left\|\frac{1}{\rho}\right\|_{L_{\infty}\left(Q_{\tau}\right)} \leqslant c
\end{aligned}
$$

əде $Q_{\tau}=\Omega \times(0, \tau)$.

ДокАЗАТЕЛЬСТво. Введем функции

$$
z(t)=\int_{\Omega}\left(F^{\delta}\left(u_{x}\right)+\frac{\rho u_{x}^{2}}{2}\right) d x, \quad y(t)=\int_{0}^{t} \int_{\Omega}\left(\rho u_{t}^{2}+f^{\delta}\left(u_{x}\right)_{x} u_{x x}\right) d x d s .
$$

Уравнение (11) влечет выполнение неравенств

$$
\begin{aligned}
\inf _{\Omega} \rho_{0}^{\delta}(x) \exp \left(-\int_{0}^{t}\left(\int_{\Omega} u_{x x}^{2} d x\right)^{1 / 2} d s\right) & \leqslant \rho(x, t) \\
& \leqslant \sup _{\Omega} \rho_{0}^{\delta}(x) \exp \left(\int_{0}^{t}\left(\int_{\Omega} u_{x x}^{2} d x\right)^{1 / 2} d s\right),
\end{aligned}
$$

из которых с учетом неравенства (6) вытекает, что

$$
c^{-1} \exp \{-c y(t)\} \leqslant \rho(x, t) \leqslant c \exp \{c y(t)\} .
$$

Кроме того, замечая, что из (11) следует уравнение $\rho D_{t}\left(v_{x}\right)=u_{x x}$, где $v=1 / \rho$, выводим соотношение

$$
\frac{d}{d t} \int_{\Omega} \frac{\rho v_{x}^{2}}{2} d x=\int_{\Omega} v_{x} u_{x x} d x
$$

Проинтегрируем его по времени и оценим с помощью неравенства Юнга

$$
\int_{\Omega} \rho v_{x}^{2} d x \leqslant c\left(1+\int_{0}^{t} \int_{\Omega} \frac{u_{x x}^{2}}{\rho} d x d s+\int_{0}^{t} \int_{\Omega} \rho v_{x}^{2} d x d s\right) .
$$

Тогда, используя лемму Гронуолла и (6), получаем неравенство

$$
\int_{\Omega} \rho v_{x}^{2} d x \leqslant c(y+1) e^{c \sqrt{y}} \leqslant c e^{c y}
$$

Умножая (12) на $u_{t}-u_{x x}$ и учитьвая $(11)$ и $(13)$, приходим к соотношению

$$
z^{\prime}(t)+y^{\prime}(t)=\int_{\Omega}\left(-R \rho_{x} u_{t}+R \rho_{x} u_{x x}+2 \rho u u_{x} u_{x x}-\rho u u_{x} u_{t}-\rho_{x} u_{t} u_{x}\right) d x
$$

Заметим, что $y^{\prime}(t) \geqslant 0$. Тогда, оценивая слагаемые в правой части этого равенства по неравенствам Гёльдера и Юнга с использованием оценок (15) и (16), вьводим неравенство

$$
z^{\prime}+y^{\prime} \leqslant \varepsilon y^{\prime}+c e^{c(z+y)}
$$


Из него, подбирая $\tau>0$ достаточно мальм, получим оценку

$$
\sup _{0 \leqslant t \leqslant \tau}(z(t)+y(t)) \leqslant c
$$

которая вместе с (15) и (16) завершает доказательство леммы.

Устремим $N \rightarrow \infty$ в уравнениях (11)-(14), Полученные в лемме 1 оценки позволяют выделить $*$-слабо сходящиеся подпоследовательности $\rho^{N} \rightarrow \rho$ в $L_{\infty}\left(0, \tau ; W_{2}^{1}(\Omega)\right)$, $u^{N} \rightarrow u$ в $L_{\infty}\left(0, \tau ; W_{2}^{1}(\Omega)\right) \cap L_{2}\left(0, \tau ; W_{2}^{2}(\Omega)\right)$. Покажем, что пара $(\rho, u)$ является решением $(7)-(10)$ на $(0, \tau)$.

Из равномерных оценок $\rho^{N}$ в $L_{\infty}\left(0, \tau ; W_{2}^{1}(\Omega)\right)$ и $\rho_{t}^{N}$ в $L_{\infty}\left(0, T ; L_{2}(\Omega)\right)$ по теореме Арцела-Асколи [10] получаем сходимость подпоследовательности

$$
\rho^{N} \rightarrow \rho \quad \text { сильно в } C\left([0, \tau], L_{2}(\Omega)\right) .
$$

Аналогично, равномерная ограниченность $u^{N}$ в $L_{\infty}\left(0, \tau ; W_{2}^{1}(\Omega)\right)$ и $u_{t}^{N}$ в $L_{2}\left(Q_{\tau}\right)$ дает возможность выделить сильно сходящуюся подпоследовательность

$$
u^{N} \rightarrow u \quad \text { сильно в } C\left([0, \tau], L_{2}(\Omega)\right) .
$$

Из ограниченности $u_{t}^{N}$ в $L_{2}\left(Q_{\tau}\right)$ следуют равномерные оценки на $u_{t x}^{N}$ в пространстве $L_{2}\left(0, \tau ; W_{2}^{-1}(\Omega)\right)$, что вместе с оценками $u_{x}^{N}$ в $L_{2}\left(0, \tau ; W_{2}^{1}(\Omega)\right)$ приводит [10] к сходимости подпоследовательности

$$
u_{x}^{N} \rightarrow u_{x} \quad \text { сильно в } L_{2}\left(Q_{\tau}\right) \text {. }
$$

Таким образом, из (17) и (18) выводим, что уравнение неразрьвности (7) и начальные условия (9) вьполнены для предельных функций $\rho$ и $u$. Переходя к пределу в (13) и учитьвая, что из (18) и (19) следует сходимость $u^{N} \rightarrow u$ сильно в $L_{2}\left(0, \tau ; W_{2}^{1}(\Omega)\right)$, доказьваем (10).

Слабая сходимость подпоследовательности $u_{t}^{N} \rightarrow u_{t}$ в $L_{2}\left(Q_{\tau}\right)$ и $(17)$ на основе метода компенсированной компактности [11] дают $*$-слабую сходимость $\rho^{N} u_{t}^{N} \rightarrow \rho u$ в $L_{2}\left(0, \tau ; L_{1}(\Omega)\right)$. Вместе с этим из ограниченности $\rho^{N} u_{t}^{N}$ в $L_{2}\left(Q_{\tau}\right)$ следует слабая сходимость $\rho^{N} u_{t}^{N} \rightarrow \rho u$ в $L_{2}\left(Q_{\tau}\right)$.

Из (17) и (19) выводим, что $\rho^{N} u_{x}^{N} \rightarrow \rho u_{x}$ сильно в $L_{2}\left(0, \tau ; L_{1}(\Omega)\right)$. Соответствующие оценки приводят к *-слабой сходимости $\rho^{N} u_{x}^{N} \rightarrow \rho u_{x}$ в пространстве $L_{\infty}\left(0, \tau ; L_{2}(\Omega)\right)$. Отсюда и из (18) получаем *-слабую сходимость $\rho^{N} u_{x}^{N} u^{N} \rightarrow \rho u_{x} u$ в $L_{\infty}\left(0, \tau ; L_{1}(\Omega)\right)$.

Учитывая $(19)$ и свойства функции $f^{\delta}(s)$, имеем *-слабую сходимость $f^{\delta}\left(u_{x}^{N}\right) \rightarrow f\left(u_{x}\right)$ в $L_{\infty}\left(0, \tau ; L_{2}(\Omega)\right)$.

Таким образом, функции $(\rho, u)$ удовлетворяют (12) при любом $1 \leqslant j<\infty$. Поскольку множество линейных комбинаций функций $\left\{\omega_{j}(x)\right\}_{1 \leqslant j<\infty}$ всюду плотно в $L_{2}(\Omega)$, то уравнение (8) выполнено почти всюду в $Q_{\tau}$.

Итак, система (7)-(10) локально по времени разрешима в следующих классах. Если $(u, \rho)$ ее решение, то уравнения $(7)-(8)$ вьполнены почти всюду в $Q_{\tau},(9)$ - почти всюду в $\Omega$, а $(10)$ - почти всюду в $\Gamma \times(0, \tau)$. Кроме того, $\rho>0$ и справедливы следуюшие включения: $u \in L_{\infty}\left(0, \tau ; W_{2}^{1}(\Omega)\right) \cap L_{2}\left(0, \tau ; W_{2}^{2}(\Omega)\right) ; \rho \in L_{\infty}\left(0, \tau ; W_{2}^{1}(\Omega)\right)$; $\rho_{t} \in L_{\infty}\left(0, \tau ; L_{2}(\Omega)\right) ; f\left(u_{x}\right)_{x}, u_{t} \in L_{2}\left(Q_{\tau}\right)$. 
4. Глобальные априорные оценки. В этом пункте строятся априорные оценки для решений $(\rho, u)$ задач (7)-(10). Отметим, прежде всего, неотрицательность плотности $\rho$ в силу того, что она, как показано в п. 3 , является пределом неотрицательных функций. Этот факт будет использоваться в доказательстве лемм 2 и 4 .

ЛЕмма 2 (энергетическая оценка). Существует такая константа с >0, что

$$
\left\|\rho u^{2}+g(\rho)\right\|_{L_{\infty}\left(0, T ; L_{1}(\Omega)\right)}+\left\|\sigma u_{x}\right\|_{L_{1}\left(Q_{T}\right)} \leqslant c
$$

əде $g(\rho)=\rho \ln \rho-\rho+1$.

ДоКАЗАТЕЛЬСТвО. Умножим уравнение импульсов (8) на $u$ и используем уравнение неразрывности (7). После интегрирования по $\Omega$ с учетом значений $u$ на $\Gamma$, получим равенство

$$
\frac{d}{d t} \int_{\Omega} \frac{\rho u^{2}}{2} d x+\int_{\Omega} \sigma u_{x} d x+R \int_{\Omega} \rho_{x} u d x=0 .
$$

Замечая, что $g^{\prime \prime}(\rho) \rho=1$, приходим к цепочке тождеств

$$
\int_{\Omega} \rho_{x} u d x=\int_{\Omega} \rho g^{\prime \prime}(\rho) \rho_{x} u d x=-\int_{\Omega}(\rho u)_{x} g^{\prime}(\rho) d x=\int_{\Omega} \rho_{t} g^{\prime}(\rho) d x=\frac{d}{d t} \int_{\Omega} g(\rho) d x .
$$

Подставляя его в предыдущее равенство и интегрируя по времени, выводим необходимую оценку.

Легко видеть, что условие 3 на рост $\partial F(s)$ приводит к оценкам для $u_{x}$ и $\sigma$ в $L_{2}\left(Q_{T}\right)$.

ЛЕмма 3. Функиия $\beta$, заданная соотношением

$$
\beta(x, t)=\int_{0}^{t}\left(\sigma(x, \tau)-R \rho(x, \tau)-\rho(x, \tau)(u(x, \tau))^{2}\right) d \tau+\int_{l}^{x} \rho_{0}(\xi) u_{0}(\xi) d \xi
$$

принадлежит $L_{\infty}\left(Q_{T}\right)$.

ДокАЗАТЕльство. Следует из теоремы вложения, поскольку функции $\beta_{x}=\rho u$ и $\beta$ по лемме 2 принадлежат пространству $L_{\infty}\left(0, T ; L_{1}(\Omega)\right)$.

ЛЕмма 4 (оценка для плотности сверху). Существует такая константа $c>0$, чmo $\rho \leqslant c$.

ДокАЗАТЕЛЬСтво. Обозначим $D_{t}=\partial_{t}+u \partial_{x}$ производную вдоль траектории. Вычислим и оценим $D_{t}\left(\rho^{K} e^{\beta}\right)$ с функцией $\beta$ из предыдущей леммы и константой $K$ из левого неравенства в условии 3

$$
D_{t}\left(\rho^{K} e^{\beta}\right)=\rho^{K} e^{\beta}\left(-K \rho u_{x}+\sigma-R \rho\right) \leqslant L \rho^{K} e^{\beta}
$$

Здесь использовалось неравенство из условия 3 и неотрищательность плотности. Тогда по лемме Гронуолла получаем оценку $\rho^{K} e^{\beta} \leqslant c$. И, наконец, применяя результат леммы 3 , приходим к неравенству $\rho \leqslant c$.

ЛЕмма 5 (оценка для плотности снизу). Существует такая константа $c>0$, чmo $\rho \geqslant c$. 
ДокАЗАтЕльство. Аналогично предыдущей лемме, найдем $D_{t}\left(\rho^{-K} e^{-\beta}\right)$ и оценим его с помощью правого неравенства из условия 3 и утверждения леммы 4

$$
D_{t}\left(\rho^{-K} e^{-\beta}\right)=\rho^{-K} e^{-\beta}\left(K \rho u_{x}-\sigma+R \rho\right) \leqslant(L+c) \rho^{-K} e^{-\beta} .
$$

Далее, используя неравенство Гронуолла, и с учетом ограниченности $\beta$ получаем требуемое.

Из лемм 2 и 5 несложно вьвести важное следствие: $u \in L_{\infty}\left(0, T ; L_{2}(\Omega)\right)$.

Следующая лемма является дополнительной априорной оценкой при соответствующих предположениях на начальную скорость.

ЛЕмма 6 (дополнительная оценка скорости). Существует константа $c>0$ такая, что справедливо неравенство

$$
\|u\|_{L_{\infty}\left(0, T ; L_{q}(\Omega)\right)}+\left\|\sigma u_{x}|u|^{q-2}\right\|_{L_{1}\left(Q_{T}\right)} \leqslant c .
$$

ДокАЗАТЕЛьСтво. Соответствующий результат при $q=2$ доказан в лемме 2. Далее применим индукцию по $q$. Предположим, что для $q-1$ соответствующее утверждение справедливо. Аналогично доказательству леммы 2 умножим уравнение (8) на $|u|^{q-1} \operatorname{sign}(u)$ и используем уравнение неразрывности $(7)$. После интегрирования по $\Omega$ с учетом значений $u$ на Г получим неравенство

$$
\frac{d}{d t} \int_{\Omega} \frac{\rho|u|^{q}}{q} d x+(q-1) \int_{\Omega} \sigma u_{x}|u|^{q-2} d x \leqslant(q-1) \int_{\Omega} R \rho u_{x}|u|^{q-2} d x .
$$

Здесь правую часть можно оценить с применением неравенства Коши и с учетом результата леммы 5 (оценки на плотность снизу)

$$
\int_{\Omega} R \rho u_{x}|u|^{q-2} d x \leqslant c\left(\left\|u_{x}^{2}|u|^{q-3}\right\|_{L_{1}(\Omega)}+\|u\|_{L_{q-1}(\Omega)}^{q-1}\right) .
$$

Так как по предположению индукции $\|u\|_{L_{q-1}(\Omega)} \in L_{\infty}(0, T)$ и $\left\|u_{x}^{2} u^{q-3}\right\|_{L_{1}(\Omega)} \in L_{1}(0, T)$, использование леммы Гронуолла завершает доказательство.

5. Сходимость приближенных решений. Для решений $\left(\rho^{\delta}, u^{\delta}\right)$ системы (7)(10) следует, в частности, выполнение следующих интегральных тождеств:

$$
\begin{aligned}
& \int_{0}^{t} \int_{\Omega} \rho^{\delta}\left(\varphi_{t}+u^{\delta} \varphi_{x}\right) d x d s=\int_{\Omega} \rho^{\delta} \varphi d x-\int_{\Omega} \rho_{0}^{\delta} \varphi(x, 0) d x \\
& \int_{0}^{t} \int_{\Omega}\left(\rho^{\delta} u^{\delta} \psi_{t}+\rho^{\delta} u^{\delta} u^{\delta} \psi_{x}+f^{\delta}\left(u_{x}^{\delta}\right) \psi_{x}-R \rho^{\delta} \psi_{x}\right) d x d s \\
& =\int_{\Omega} \rho^{\delta} u^{\delta} \psi d x-\int_{\Omega} \rho_{0}^{\delta} u_{0}^{\delta} \psi(x, 0) d x
\end{aligned}
$$

для почти всех $t \in(0, T)$ и для всех $\varphi, \psi \in C^{\infty}\left(Q_{T}\right)$, равных нулю на $\Gamma$.

Из лемм $2-5$ следует, что $\rho^{\delta}$ и $1 / \rho^{\delta}$ равномерно ограничены в $L_{\infty}\left(Q_{T}\right)$ и $u^{\delta}-$ в $L_{\infty}(0$, $\left.T ; L_{2}(\Omega)\right)$ и $L_{2}\left(0, T ; W_{2}^{1}(\Omega)\right)$. Это позволяет, устремляя $\delta \rightarrow 0$, выделить $*$-слабо сходящиеся подпоследовательности $\rho^{\delta} \rightarrow \rho$ в $L_{\infty}\left(Q_{T}\right)$ (причем $\rho \geqslant c>0$ ), а $u^{\delta} \rightarrow u$ 
в $L_{\infty}\left(0, T ; L_{2}(\Omega)\right) \cap L_{2}\left(0, T ; W_{2}^{1}(\Omega)\right)$. Последнее утверждение влечет непрерьвность $u$ для почти всех $t \in(0, T)$ как функции от $x$ и равенство $u=0$ на $\Gamma$ для почти всех $t \in(0, T)$.

Сходимость $\rho^{\delta} \rightarrow \rho *$-слабо в $L_{\infty}\left(Q_{T}\right)$ и ограниченность $\rho_{t}^{\delta}=-\left(\rho^{\delta} u^{\delta}\right)_{x}$ в пространстве $L_{\infty}\left(0, T ; W_{2}^{-1}(\Omega)\right)$ позволяют по теореме Арцела-Асколи выделить сильно сходящуюся в $C\left([0, T] ; W_{2}^{-1}(\Omega)\right)$ подпоследовательность $\rho^{\delta} \rightarrow \rho$. В частности, равномерно по $t \in(0, T)$ для любой $\varphi \in C_{0}^{\infty}(\Omega)$ имеет место сходимость

$$
\int_{\Omega} \rho^{\delta}(t) \varphi d x \rightarrow \int_{\Omega} \rho(t) \varphi d x
$$

Используя ограниченность $\rho^{\delta}$ в $L_{\infty}(\Omega)$ для почти всех $t \in(0, T)$, получаем сходимость $\rho^{\delta}(t) \rightarrow \rho(t) *$-слабо в $L_{\infty}(\Omega)$ для почти всех $t \in(0, T)$.

Как было отмечено, подпоследовательность $\rho^{\delta} \rightarrow \rho$ сильно в пространстве $C([0, T]$; $\left.W_{2}^{-1}(\Omega)\right)$, а $u^{\delta} \rightarrow u$ слабо в $L_{2}\left(0, T ; W_{2}^{1}(\Omega)\right)$. Применение метода компенсированной компактности приводит к *-слабой сходимости подпоследовательности $\rho^{\delta} u^{\delta} \rightarrow \rho u$ в $L_{2}\left(0, T ; L_{1}(\Omega)\right)$. Тогда из равномерной ограниченности $\rho^{\delta} u^{\delta}$ в пространстве $L_{\infty}(0, \tau$; $\left.L_{2}(\Omega)\right)$ следует, что $\rho^{\delta} u^{\delta} \rightarrow \rho u *$-слабо в $L_{\infty}\left(0, T ; L_{2}(\Omega)\right)$.

Из *-слабой сходимости $\rho^{\delta} u^{\delta} \rightarrow \rho u$ в $L_{\infty}\left(0, T ; L_{2}(\Omega)\right)$ и равномерной ограниченности $\left(\rho^{\delta} u^{\delta}\right)_{t}=\left(f^{\delta}\left(u_{x}^{\delta}\right)-R \rho^{\delta}-\left(\rho^{\delta} u^{\delta} u^{\delta}\right)\right)_{x}$ в $L_{2}\left(0, T ; W_{2}^{-1}(\Omega)\right)$ по теореме Арцела-Асколи получаем сильную сходимость подпоследовательности $\rho^{\delta} u^{\delta} \rightarrow \rho u$ в $C\left([0, T] ; W_{2}^{-1}(\Omega)\right)$. Тогда, аналогично доказанному для $\rho^{\delta}(t)$, заключаем, что для почти всех $t \in(0, T)$ подпоследовательность $\rho^{\delta}(t) u^{\delta}(t) \rightarrow \rho(t) u(t)$ слабо в $L_{2}(\Omega)$.

Далее, сильная сходимость $\rho^{\delta} u^{\delta} \rightarrow \rho u$ в $C\left([0, T] ; W_{2}^{-1}(\Omega)\right)$ и слабая $-u^{\delta} \rightarrow u$ в $L_{2}\left(0, T ; W_{2}^{1}(\Omega)\right)$ на основе компенсированной компактности дает слабую сходимость подпоследовательности $\rho^{\delta} u^{\delta} u^{\delta} \rightarrow \rho u u$ в $L_{2}\left(0, T ; L_{1}(\Omega)\right)$. Более того, соответствующие равномерные оценки приводят к слабой сходимости $\rho^{\delta} u^{\delta} u^{\delta} \rightarrow \rho u u$ в $L_{\infty}\left(0, T ; L_{1}(\Omega)\right) \cap$ $L_{2}\left(Q_{\tau}\right)$.

Подпоследовательность $f^{\delta}\left(u_{x}^{\delta}\right) *$-слабо сходится в $L_{2}\left(Q_{T}\right)$ к некоторой функции $\sigma$. Поскольку последовательности $\rho_{0}^{\delta} \rightarrow \rho_{0}$ и $u_{0}^{\delta} \rightarrow u_{0}$ сильно в $L_{2}(\Omega)$, переходя к пределу в (20) и (21), получаем следующие интегральные тождества:

$$
\begin{aligned}
\int_{0}^{t} \int_{\Omega}\left(\rho \varphi_{t}+\rho u \varphi_{x}\right) d x d s & =\int_{\Omega} \rho \varphi d x-\int_{\Omega} \rho_{0} \varphi(x, 0) d x \\
\int_{0}^{t} \int_{\Omega}\left(\rho u \psi_{t}+\rho u u \psi_{x}+\sigma \psi_{x}-R \rho \psi_{x}\right) d x d s & =\int_{\Omega} \rho u \psi d x-\int_{\Omega} \rho_{0} u_{0} \psi(x, 0) d x
\end{aligned}
$$

Осталось показать справедливость включения (5). Оно равносильно неравенству

$$
\sigma\left(w-u_{x}\right) \leqslant F(w)-F\left(u_{x}\right)
$$

для всех $w \in \mathbb{R}$ почти всюду в $Q_{T}$.

Очевидно, что для приближенных решений вьполнено неравенство

$$
f^{\delta}\left(u_{x}^{\delta}\right)\left(w-u_{x}^{\delta}\right) \leqslant F^{\delta}(w)-F^{\delta}\left(u_{x}^{\delta}\right)
$$


умножая котороена бесконечно дифференцируемую неотрицательную функцию $\xi$ и проинтегрировав по $Q_{T}$, получим

$$
\int_{0}^{T} \int_{\Omega} \xi f^{\delta}\left(u_{x}^{\delta}\right)\left(w-u_{x}^{\delta}\right) d x d t \leqslant \int_{0}^{T} \int_{\Omega} \xi\left(F^{\delta}(w)-F^{\delta}\left(u_{x}^{\delta}\right)\right) d x d t
$$

Заметим, что из оценок для $\rho$ в $W_{\infty}^{1}\left(0, T ; W_{2}^{-1}(\Omega)\right) \cap L_{\infty}\left(Q_{T}\right), \rho u$ в $L_{\infty}\left(0, T ; L_{2}(\Omega)\right)$ и для почти всех $t \in(0, T) \quad \rho$ в $L_{\infty}(\Omega)$ вытекает справедливость тождества $(22)$ для $\varphi$ из $L_{1}\left(0, T ; W_{2}^{1}(\Omega)\right) \cap L_{\infty}\left(0, T ; L_{1}(\Omega)\right)$ с условием $\varphi=0$ на $\Gamma$. В частности, можно взять в качестве $\varphi$ функцию $u^{2} / 2$.

Включение $u \in L_{\infty}\left(0, T ; L_{2}(\Omega)\right) \cap L_{2}\left(0, T ; W_{2}^{1}(\Omega)\right)$ дает оценку на $u$ в пространстве $L_{4}\left(Q_{T}\right)$, которая приводит к ограниченности $\rho u u$ в $L_{2}\left(Q_{T}\right)$. Тогда из включений $\rho u \in W_{2}^{1}\left(0, T ; W_{2}^{-1}(\Omega)\right), \rho u u \in L_{2}\left(Q_{T}\right), \rho \in L_{\infty}\left(Q_{T}\right), \sigma \in L_{2}\left(Q_{T}\right)$ и для почти всех $t \in(0, T), \rho u \in L_{2}(\Omega)$ следует, что $(23)$ выполнено и для всех $\psi \in L_{2}\left(0, T ; W_{2}^{1}(\Omega)\right) \cap$ $L_{\infty}\left(0, T ; L_{2}(\Omega)\right)$ таких, что $\psi=0$ на $\Gamma$. Это позволяет взять $\psi=u$.

Подставляя в $(22) \varphi=u^{2} / 2$ и в $(23) \psi=u$ и вычитая первое из второго, получаем соотношение

$$
\int_{0}^{T} \int_{\Omega}\left(\sigma u_{x}-R \rho u_{x}\right) d x d s=\int_{\Omega} \frac{\rho u^{2}}{2} d x-\int_{\Omega} \frac{\rho_{0} u_{0}^{2}}{2} d x .
$$

Производя аналогичные операции с (20) и (21) и переходя к пределу при $\delta \rightarrow 0$, приходим к равенству

$$
\lim _{\delta \rightarrow 0} \int_{0}^{T} \int_{\Omega} f^{\delta}\left(u_{x}^{\delta}\right) u_{x}^{\delta} d x d \tau-\int_{0}^{T} \int_{\Omega} R \rho u_{x} d x d s=\int_{\Omega} \frac{\rho u^{2}}{2} d x-\int_{\Omega} \frac{\rho_{0} u_{0}^{2}}{2} d x .
$$

Сравнивая его с предыдущим, вьводим соотношение

$$
\lim _{\delta \rightarrow 0} \int_{0}^{T} \int_{\Omega} f^{\delta}\left(u_{x}^{\delta}\right) u_{x}^{\delta} d x d \tau=\int_{0}^{T} \int_{\Omega} \sigma u_{x} d x d \tau
$$

Наконец, из свойств функций $F^{\delta}(s)$ следует, что

$$
\int_{0}^{T} \int_{\Omega} \xi\left|F^{\delta}\left(u_{x}^{\delta}\right)-F\left(u_{x}^{\delta}\right)\right| d x d t \leqslant \tau M \delta \operatorname{meas}(\Omega)\|\xi\|_{C\left(Q_{T}\right)} \rightarrow 0 \quad \text { при } \delta \rightarrow 0 .
$$

С другой стороны, функционал $\Phi(v)$, определенньй соотношением

$$
\Phi(v)=\int_{0}^{T} \int_{\Omega} \xi F(v) d x d t,
$$

является непрерьвным и выпуклым в $L_{2}\left(Q_{T}\right)$. Так как $L_{2}\left(Q_{T}\right)$ - рефлексивное пространство, то $\Phi(v)$ слабо полунепрерывен снизу; в частности, так как $u_{x}^{\delta} \rightarrow u_{x}$ слабо в $L_{2}\left(Q_{T}\right)$, то

$$
\Phi\left(u_{x}\right) \leqslant \liminf \Phi\left(u_{x}^{\delta}\right) .
$$

Отсюда, из (25) и (26) вытекает выполнение неравенства

$$
\int_{0}^{T} \int_{\Omega} \xi \sigma\left(w-u_{x}\right) d x d t \leqslant \int_{0}^{T} \int_{\Omega} \xi\left(F(w)-F\left(u_{x}\right)\right) d x d t .
$$

В силу произвольности и неотрицательности функции $\xi$ получаем (24) почти всюду в $Q_{T}$.

Справедливость теоремы установлена. Необходимые априорные оценки для решений задач (1)-(5) верны, поскольку они являются пределами решений задач (7)-(10). Кроме того, заменяя нумерацию формул в доказательствах лемм 2-6, все априорные оценки выводятся непосредственно. 
ЗАМЕЧАниЕ. При наличии массовых сил $\Phi(x, t)$ уравнение импульсов $(2)$ модифицируется:

$$
\rho\left(u_{t}+u u_{x}\right)=\sigma_{x}-R \rho_{x}+\rho \Phi .
$$

Сходным образом изменяется интегральное тождество в определении обобщенного решения. Аналог сформулированной вьше теоремы имеет место, например, при $\Phi \in$ $L_{\infty}\left(Q_{T}\right)$.

Автор очень признателен профессору А. В. Кажихову за поддержку и внимание и рецензенту за полезные замечания.

\section{СПИСОК ЦИТИРОВАННОЙ ЛИТЕРАТУРЫ}

[1] Truesdell C. A First Course in Rational Continuum Mechanics. General Concepts (2nd edition). V. 1. San Diego-London: Academic Press, 1991.

[2] Антонцев С.Н., Кажихов А. В., Монахов В.Н. Краевые задачи механики неоднородных жидкостей. Новосибирск: Наука, 1983.

[3] Вайгант В. А. Неоднородные задачи для уравнений вязкого теплопроводного газа // Математические проблемы механики сплошных сред. Динамика сплошной среды. Вып. 97. Новосибирск: Ин-т Гидродинамики СО АН СССР, 1990. С. 3-21.

[4] Амосов А. А., Злотник А. А. Разрешимость "в целом" одного класса квазилинейных систем уравнений составного типа с негладкими данными // Дифференц. уравнения. 1994. T. $30(4)$. C. $596-609$.

[5] Вайгант В. А., Кажихов А. В. О существовании глобальных решений двумерных уравнений Навье-Стокса сжимаемой вязкой жидкости // Сиб. матем. ж. 1995 . Т. $36(6)$. C. $1283-1316$.

[6] Málek J., Necas J., Rokyta M., Růžička M. Weak and measure-valued solutions to evolutionary PDEs, Applied Mathematics and Mathematical Computation, 13. London: Chapman \& Hall, 1996.

[7] Мамонтов A. E. Orlicz spaces in the existense problem of global solutions to viscous compressible nonlinear fluid equations. Препринт СО РАН № 2-96. Новосибирск: Ин-т гидродинамики, 1996.

[8] Showalter W. R. Mechanics of non-Newtonian fluids. Oxford: Pergamon Press, 1978.

[9] Shelukhin V. V. A shear flow problem for the compressible Navier-Stokes equations // Internat. J. Non-Linear Mech. 1998. V. 33 (2). P. 247-257.

[10] Simon J. Compact sets in the space $L_{p}(0, T ; B) / /$ Ann. Matematica Pura ed Applicata (IV). 1987. V. VCXLVI. P. 65-69.

[11] Tartar L. Compensated compactness and application to P.D.E. // Nonlinear Analysis and Mechanics, 4 (Heriot-Watt Symposium) / ed. R. J. Knops. Pitman Reseach Notes in Math. Series. V. 39, 1979. P. 136-212.

Институт гидродинамики им. М. А. Лаврентьева СО РАН

Поступило

E-mail: karhikhov@hydro.usc.ru

01.06 .1998

Исправленный вариант

10.05 .1999 